\title{
激光/红外共孔径无热化紧凑型光学系统设计
}

\author{
贺祥清 ${ }^{1,2}$ ，廖小军 ${ }^{1,2}$ ，段 媛 ${ }^{1,2}$, 张影烨 1,2
}

(1. 四川长九光电科技有限责任公司, 四川 绵阳 621000；2. 四川九洲电器集团有限责任公司，四川 绵阳 621000）

\begin{abstract}
摘要: 基于激光测距和红外目标探测需求, 设计了激光/红外共孔径无热化紧凑型光学系统。系统参数 设计如下: 工作波段为 $1.064 \mu \mathrm{m}$ 激光和 $7.7 \sim 9.3 \mu \mathrm{m}$ 长波红外, 入瞳直径均为 $120 \mathrm{~mm}$; 激光焦距为 $800 \mathrm{~mm}$; 长波红外焦距为 $240 \mathrm{~mm}, \mathrm{~F}$ 数为 2 , 视场为 $2.29^{\circ} \times 1.83^{\circ}$ 。选择带有 Ritchey-Chretien (RC) 反射系统的折反式光学布局, 缩短系统纵向尺寸。光学系统共用主镜和次镜, 利用次镜实现激光和红 外分光。长波红外采用二次成像结构, 达到 $100 \%$ 冷光阑效率。通过选择合适的光学材料、结构材料 和合理分配光焦度, 实现了光学被动式消热差。在 $-50^{\circ} \mathrm{C} \sim+70^{\circ} \mathrm{C}$ 范围内, 激光接收能量集中度高, 长波红外成像质量良好, 满足实际使用需求。
\end{abstract}

关键词: 光学设计; 激光测距; 红外成像; 共孔径; 被动式消热差; 紧凑型

中图分类号：TN216 文献标识码：A 文章编号：1001-8891(2020)05-0461-07

\section{Common Aperture and Athermalization Design of Compact Laser/Infrared Optical System}

\author{
HE Xiangqing ${ }^{1,2}$, LIAO Xiaojun ${ }^{1,2}$, DUAN Yuan ${ }^{1,2}$, ZHANG Haoye ${ }^{1,2}$ \\ (1. Sichuan C\&J Electrooptical Technology Co., Ltd., Mianyang 621000, China; \\ 2. Sichuan Jiuzhou Electric Group Co., Ltd., Mianyang 621000, China)
}

\begin{abstract}
Based on requirements of laser ranging and infrared target detection, a laser/ infrared compact optical system with a common aperture and athermalization is designed. The parameters of the optical system are defined as follows: the operating waveband covers $1.064 \mu \mathrm{m}$ of laser and 7.7-9.3 $\mu \mathrm{m}$ of wavelength infrared radiation (LWIR), the diameters of the two entrance pupils are $120 \mathrm{~mm}$; the focal length of the laser is $800 \mathrm{~mm}$; the focal length of the LWIR is $240 \mathrm{~mm}$, the F-number is 2, the field of view is $2.29^{\circ} \times 1.83^{\circ}$. the vertical size of system is shortened by utilizing a catadioptric optical system with RC. The laser system shares the primary mirror and secondary mirror with the LWIR system. The beam splitting of the laser and LWIR is achieved by the secondary mirror. By using the double imaging configuration, the LWIR system exhibits nearly $100 \%$ efficiency of the cold stop. Optical passive athermalization is realized by choosing the appropriate optical materials, mechanical materials and assigning the optical power. In the temperature range of $-50^{\circ} \mathrm{C}$ to $70^{\circ} \mathrm{C}$, the energy concentration of the laser receiving optical system is extremely high, and the image quality of the LWIR system is also quite good, which satisfies the requirements of practical applications.
\end{abstract}

Key words: optical design, laser ranging, infrared imaging, common aperture, passive athermalization, compact

\section{0 引言}

红外搜索跟踪（Infrared search and track, IRST) 系统在夜视、高精度跟踪和反隐身等方面具有明显的 优势, 已成为高技术军事装备发展的一个重点 ${ }^{[1-2]}$ 。红 外搜索跟踪系统常配置红外热像仪和激光测距机等
光电传感器, 可对目标进行搜索、截获、跟踪和激光 测距, 以实现对军事目标的精确打击。因此, 世界主 要军事强国的机载、车载和舰载等武器装备都配备了 激光测距和红外成像系统。

$1.064 \mu \mathrm{m}$ 和 $1.57 \mu \mathrm{m}$ 激光测距技术经过多年的发 展, 已成熟并在军事上得到广泛的应用。红外成像系 
统是通过收集目标的红外辐射而实现对目标的探测。 红外辐射有中波 3 $5 \mu \mathrm{m}$ 和长波 8 $12 \mu \mathrm{m}$ 两个大气 窗口，3 $5 \mu \mathrm{m}$ 波段一般用于搜索温差较大的目标， 8 $12 \mu \mathrm{m}$ 一般用于搜索温差较小的目标, 中波和长波 红外具有各自的优点, 可根据目标特性和具体的使用 条件进行合理选择 ${ }^{[3]}$ 。

基于红外搜索跟踪系统在激光测距和红外目标 探测方面的需要, 激光/红外复合光学系统得到了高度 重视。文献[4]报道了激光/红外共光路无热化光学系 统设计, 该系统采用 $1.57 \mu \mathrm{m}$ 激光和 3 $5 \mu \mathrm{m}$ 中波共 光路, 中波红外焦距为 $150 \mathrm{~mm}, F$ 数为 2 , 全视场为 $4.2^{\circ}$, 在 $-50^{\circ} \mathrm{C} \sim+70^{\circ} \mathrm{C}$ 范围内实现了无热化设计。 文献[5]报道了中波红外/激光双模导引头光学系统小 型化设计, 采用 3 $5 \mu \mathrm{m}$ 中波和 $1.06 \mu \mathrm{m}$ 激光共光路, 中波红外入瞳直径为 $75 \mathrm{~mm}$, 焦距为 $150 \mathrm{~mm}$, 全视 场为 $4^{\circ}$; 激光发射光学系统焦距为 $16.67 \mathrm{~mm}$, 口径为 $7.52 \mathrm{~mm}$; 激光接收系统焦距为 $130 \mathrm{~mm}$, 口径为 $75 \mathrm{~mm}$; 该系统采用卡塞格林结构, 易于实现小型化。

本文根据实际的使用需求, 设计了一套激光/红 外共孔径无热化紧凑型光学系统, 工作波段为激光 $1.064 \mu \mathrm{m}$ 和长波 $7.7 \sim 9.3 \mu \mathrm{m}$, 激光选用 $\mathrm{APD}$ (Avalanche Photodiode) 探测器, 长波红外选用 320 $\times 256$ 像元制冷型探测器。该系统采用共孔径设计, 实现大口径、大视场探测目标, 具有结构紧凑、体积 小、重量轻等优点; 进行无热化设计, 保证系统在一 $50^{\circ} \mathrm{C} \sim+70^{\circ} \mathrm{C}$ 温度范围内, 以实现 $1.064 \mu \mathrm{m}$ 激光测距 和 $7.7 \sim 9.3 \mu \mathrm{m}$ 红外成像。

\section{1 设计原理}

为设计出满足要求的激光/红外共孔径光学系统, 在光学系统结构形式、冷光阑匹配、无热化等方面进 行了设计。

\section{1 光学系统结构形式}

为缩短光学系统的总长度, 实现紧凑型激光/红外 共孔径光学系统, 光学系统结构的选择就变得至关重 要 ${ }^{[6]}$ 。常用光学系统主要有以下几种结构形式: 折射 式、反射式和折反式。折射式系统是最常用的光学系 统, 可以达到较大的视场和相对孔径, 但折射系统透 射的红外材料较少, 在设计激光/红外这种间隔较大的 双波段大口径光学系统时, 校正二级色差难度较大。 反射式系统一般有共轴反射和离轴三反结构, 共轴反 射视场较小, 且有中心遮挡, 离轴反射系统的视场比 共轴反射系统大, 但其像差校正和加工、装调难度较 大。折反式系统是将反射镜和校正透镜组相结合的光 学系统, 为了得到较大的视场和校正球差及彗差,
Ritchey 和 Chretien 提出了改进的卡塞格林式系统, 即 $\mathrm{RC}$ 系统 ${ }^{[7]}$, 根据设计需要可在像面之前加入校正透镜 组。比较这 3 种结构形式的优缺点, 本文选择带有 RC 反射系统的折反式光学系统。

本光学系统采用激光接收和长波红外接收在 $\mathrm{RC}$ 反射系统前端望远部分共光路的方式, 系统结构紧 凑, 实用性较强, 激光红外波段共用 RC 反射系统, 之后由分色镜分成激光和长波红外两个波段, 两波段 再采用折射式系统, 分别校正像差。

\section{2 实现 $100 \%$ 冷光阑效率}

长波红外光学系统采用 $320 \times 256$ 制冷探测器 件, 为了对杂散热辐射的抑制, 必须考虑探测器的 冷光阑 ${ }^{[8]}$ 。光瞳衔接原则决定了光学系统的出瞳位置 和大小, 为满足 $100 \%$ 冷光阑效率, 需保证光学系统 的出瞳与探测器冷光阑相匹配。大视场成像系统的有 效焦距通常较短, 为了保证足够的工作距离, 可采用 二次成像构型 ${ }^{[9]}$ 。一方面, 一次成像系统为了实现冷 光阑匹配, 必然使得制冷型红外光学系统的径向口径 过大，因此为了减小系统径向尺寸，可在一次像面后 方追加二次成像结构。另一方面, 二次成像结构对于 校正光学像差会相对容易, 光学系统第一组态负责系 统的光焦度, 第二组态负责二次成像, 并配合平衡残 余像差。

\section{3 无热化设计}

当环境温度发生变化时, 光学元件的尺寸 (曲率 半径、厚度）和折射率以及光学元件之间的间隔均会 发生改变, 这些变化会导致不同温度条件下的成像质 量变差, 因为需要对光学系统进行消热差 ${ }^{[10-11]}$ 。目前 消热差的方法主要有以下 3 种: (1)机械主动式补偿; (2)机械被动式补偿; (3)光学被动式补偿。机械主动式 和机械被动式是两种比较传统的方法, 但是这两种方 法都引入了补偿装置, 系统重、体积大、可靠性和环 境稳定性也不好。而光学被动式方法利用光学材料及 结构材料热特性之间的差异进行相互补偿来实现无 热化设计, 具有结构简单、尺寸小、重量轻, 系统可 靠性高等优点。本文系统要求做到结构紧凑和体积 小, 所以选用光学被动补偿的方式。

透镜光焦度随温度变化表示为 ${ }^{[12]}$ :

$$
\Delta \phi=\phi\left(\alpha_{\mathrm{g}}-\frac{1}{n-1} \frac{\mathrm{d} n}{\mathrm{~d} t}\right) \Delta t
$$

式中: $\phi$ 为透镜的光焦度; $\alpha_{\mathrm{g}}$ 为透镜材料的热膨胀系 数; $\mathrm{d} n / \mathrm{d} t$ 为透镜材料的折射率温度系数。多数情况下, 焦距随温度变化而减小, 公式常写成 $\Delta \phi=\delta \phi \Delta t, \delta$ 为 透镜材料的光热膨胀系数: 


$$
\delta=\alpha_{\mathrm{g}}-\frac{1}{n-1} \frac{\mathrm{d} n}{\mathrm{~d} t}
$$

光学系统相对像面的焦移需考虑镜筒随温度的 变化:

$$
\Delta f=\left(\delta+\alpha_{\text {mount }}\right) \frac{1}{\phi} \Delta t
$$

式中: $\Delta f$ 为光学系统焦距的变化量; $\alpha_{\text {mount }}$ 为结构材 料的线膨胀系数。

这里 Ritchey-Chretien 作为物镜组, 主、次镜采 用相同的基底材料, 支撑结构件选用与其热膨胀系数 相同或相近的材料, 保证光学零件和机械零件均匀地 膨胀和收缩, 则主次镜的热离焦量接近零。

目镜组的消热化设计必须同时满足光焦度、消色 差和消热差的要求, 通常需要 3 种不同材料的组合才 能实现光学消热。假设 3 片镜由一片单透镜和一组双 胶合透镜组成, 那么必须满足以下 3 个方程:

$$
\begin{gathered}
h_{1} \phi_{1}+h_{2} \phi_{2}+h_{3} \phi_{3}=h_{1} \Phi \\
h_{1}^{2} \phi_{1} / v_{1}+h_{2}^{2} \phi_{2} / v_{2}+h_{3}^{2} \phi_{3} / v_{3}=0 \\
h_{1}^{2} \phi_{1} \delta_{1}+h_{2}^{2} \phi_{2} \delta_{2}+h_{3}^{2} \phi_{3} \delta_{3}=\Phi \alpha_{\text {mount }}
\end{gathered}
$$

式中: $h_{1} 、 h_{2} 、 h_{3}$ 为第一近轴光线在各透镜的高度; $\phi_{1} 、 \phi_{2} 、 \phi_{3}$ 为透镜的光焦度; $\Phi$ 为系统的总光焦度; $v_{1} 、 v_{2} 、 v_{3}$ 为光学材料的阿贝数; $\delta_{1} 、 \delta_{2} 、 \delta_{3}$ 为光学材 料的光热膨胀系数。设计时, 先通过求解方程组得到 系统的初始结构, 然后利用光学设计软件对其进行优 化设计, 得到消热差激光和长波红外光学系统的最终 结构参数。

\section{2 光学系统设计}

\section{1 技术指标}

根据激光测距距离和红外探测距离要求, 计算了 激光接收和长波红外光学系统的技术指标, 见表 1 。

表 1 光学系统的技术指标

Table 1 Technological specifications of optical system

\begin{tabular}{lll}
\hline Parameters & Laser receiving & LWIR \\
\hline Wavelength range $/ \mu \mathrm{m}$ & 1.064 & $7.7-9.3$ \\
\hline Entrance pupil diameter & 120 & 120 \\
\hline Focal length $/ \mathrm{mm}$ & 800 & 240 \\
\hline F-number & - & 2 \\
\hline Field of view $/{ }^{\circ}$ & \pm 0.03 & $2.29 \times 1.83$ \\
\hline Detector & APD & $320 \times 256$ \\
\hline Pixel size $/ \mu \mathrm{m}$ & $\phi 800$ & $30 \times 30$ \\
\hline Temperature range $/{ }^{\circ} \mathrm{C}$ & $-500^{\circ} \mathrm{C}-+70^{\circ} \mathrm{C}$ & $-50{ }^{\circ} \mathrm{C}-+70^{\circ} \mathrm{C}$ \\
\hline
\end{tabular}

\section{2 系统设计}

2.2.1 光路布局
由于激光红外共孔径光学系统要求结构尺寸尽 量紧凑, 本文选择带有 RC 反射系统的折反式光学系 统, 结合光学系统设计结果, 采取 “ $\Pi$ ” 型布局。将 长波红外光学系统通过两个平面反射镜进行两次折 转, 成像于 RC 反射系统的侧面; 激光接收光路经主、 次镜反射后, 激光接收焦面会聚于主反射镜背面。采 用光机结构一体化设计, 将激光接收和长波红外光路 的光学零件和探测器组件安装在同一基座上, 精密调 校两个光路的主光轴, 保证了两个光轴的一致性, 也 提高了光学系统的环境适应能力。

\subsection{2 光学材料选择}

$1.064 \mu \mathrm{m}$ 激光接收波段, 可用的光学玻璃材料比 较多, 设计自由度大, 像差校正相对容易。本文选取 K9、SF3、SF4 进行激光接收光学系统的像差校正。

在长波红外波段 (8 $12 \mu \mathrm{m})$ 可用的光学材料非 常有限且价格昂贵, 这大大增加了长波红外光学系统 的像差校正难度，同时也限制了光学系统设计时的自 由度。长波波段常用的红外材料有 GaAs、Ge、ZnS、 ZnSe、AMTIR1、 $\mathrm{CaF}_{2}$ 等。考虑到材料的化学稳定性、 光加性能和镀膜性能, 本文选取 Ge、ZnS、ZnSe、 AMTIR1 进行长波红外光学系统的像差校正, 其光学 性能 ${ }^{[12]}$ 见表 2 。

\section{表 2 红外材料的光学性能}

Table 2 Performance of infrared materials

\begin{tabular}{llll}
\hline Material & Spectral range $/ \mu \mathrm{m}$ & $v_{8-12 \mu \mathrm{m}}$ & $\delta\left(\times 10^{-6}\right)$ \\
\hline $\mathrm{Ge}$ & $2.0-17.0$ & 861.03 & 124.87 \\
ZnS & $0.37-14.0$ & 22.76 & 26.17 \\
ZnSe & $0.22-20.0$ & 57.84 & 34.12 \\
AMTIR1 & $0.7-14.0$ & 119.97 & 33.47 \\
\hline
\end{tabular}

2.2.3 光学系统设计

光学系统如图 1 所示, 采用 “川” 型布局, 引入 长波折叠反射镜缩短了系统的纵向尺寸，使得系统的 整体结构更加紧凑, 满足安装尺寸要求。光学系统前 端为改进的卡塞格林反射系统, 即 RC 系统, 主镜尺 寸较大, 为方便光学加工和镀膜, 主镜材料选择融石 英, 次镜材料为 $\mathrm{Ge}$, 次镜同时承担了分色镜的作用, 次镜的第一面反射 $1.064 \mu \mathrm{m}$ 激光, 透射长波红外, 镀 制长波通分色膜。经过次镜分色后, 激光接收和长波 红外分为两个光路, 便于校正光学像差。

长波红外光学系统由前端望远系统和后端红外 成像镜头组成。前端望远系统包含主镜、次镜组和一 片准直透镜。其中次镜组第一个透镜 Ge 的第一表面 作为激光/红外分色面, 反射激光, 透射长波, 次镜组 采用两片式结构, 对系统光焦度的贡献分别为负、正, 采用 Ge 和 AMTIR1 两种材料校正中间像面的像差。 


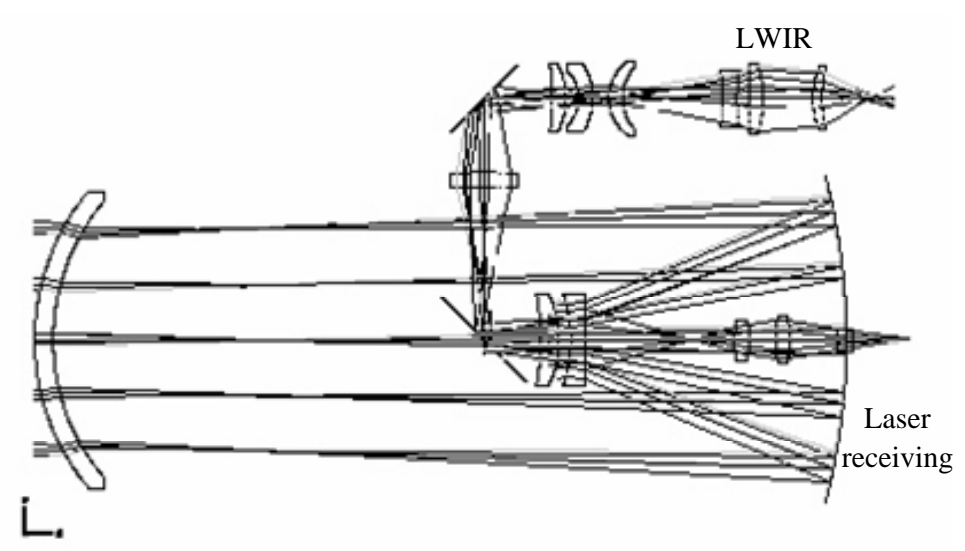

图 1 光学系统结构图

Fig.1 Structure diagram of optical system

由于残余像差不大, 准直透镜使用一片球面 Ge 透镜 可以实现系统的准直。望远系统采用光机配合消热设 计，结构件尽量选用低膨胀率的材料。

为了达到 $100 \%$ 冷光阑效率, 后端红外成像镜头 采用二次成像结构。对系统在 $-50^{\circ} \mathrm{C} \sim 70^{\circ} \mathrm{C}$ 温度范围 内, 分析长波红外光路的离焦量与温度的关系。通过 合理分配光焦度、选用合适的光学材料和结构材料、 使用非球面等方法来平衡像差和实现红外镜头的光 学被动消热。前三片镜的材料为 AMTIR1、ZnS、 AMTIR1, 对光焦度的贡献为正、负、正, 初步校正 一次像面的像差。后三片镜的材料分别为 $\mathrm{ZnS} 、 \mathrm{ZnSe}$ 、 $\mathrm{Ge}$, 光焦度的贡献为负、正、正, 负责校正系统色差 和热差, 其中靠近像面的 $\mathrm{Ge}$ 透镜的第二个面为偶次 高阶非球面, 有利于校正像差。

激光接收光学系统由主镜、次镜、二次成像镜组 组成。采用二次成像结构, 目的是在主、次镜形成的 一次像面处设置消杂光光阑, 防止杂光进入。二次成 像镜组材料为常用的光学玻璃 K9、SF3 和 SF4, 其中 SF3 透镜第一表面为偶次高阶非球面。由于光学玻璃 的折射率和线膨胀系数随温度变化不敏感, 易于实现 消热化设计。

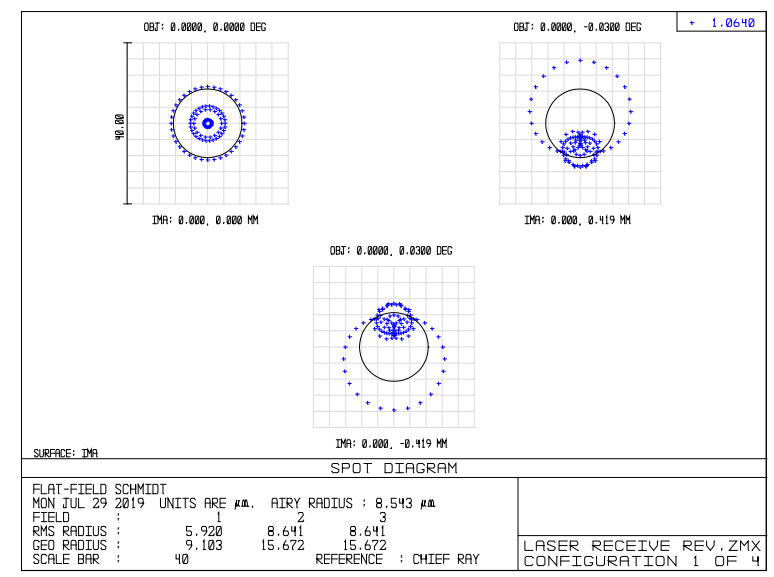

(a) $20^{\circ} \mathrm{C}$
在光学系统设计中引入非球面, 可提高光学系统 的成像质量、减小光学元件的数量。为了减小光学系 统的装配公差, 并兼顾加工成本, 非球面通常设置在 入射角度和出射角度较小的位置。为了降低光学元件 的偏心公差, 保证光学系统的装配精度和成像质量, 设计时只在光学元件的一个表面使用了非球面。

\section{3 像质评价}

\subsection{1 激光接收}

\section{1）点列图}

按照几何光学的观点, 点列图中点的分布可以近 似地代表像点的能量分布, 利用这些点的密集程度能 够衡量光学系统成像质量的好坏。均方根半径值反映 了光能的集中程度, 与几何最大半径值相比, 更能反 映光学系统的成像质量。如果点列图的均方根半径值 接近或小于艾里斑半径, 则光学系统接近衍射极限。 设计的激光接收光学系统点列图如图 2 所示。

从图 2 可以看出, 在 $-50^{\circ} \mathrm{C} \sim 70^{\circ} \mathrm{C}$ 范围内, 激光 接收光学系统的弥散斑半径均方根值接近艾里斑半 径, 弥散斑在 APD 探测器光敏面直径 $0.8 \mathrm{~mm}$ 范围内, 成像质量接近衍射极限, 消热差后光学系统像质满足 设计要求。

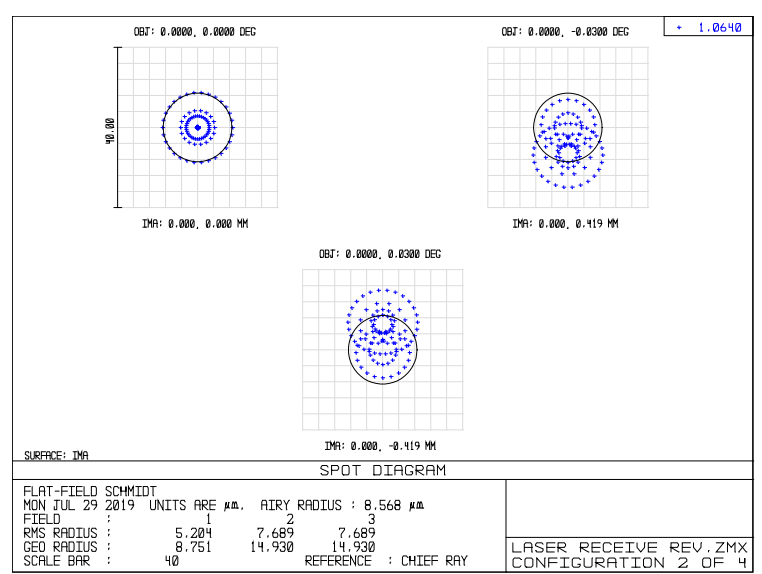

(b) $-50^{\circ} \mathrm{C}$ 


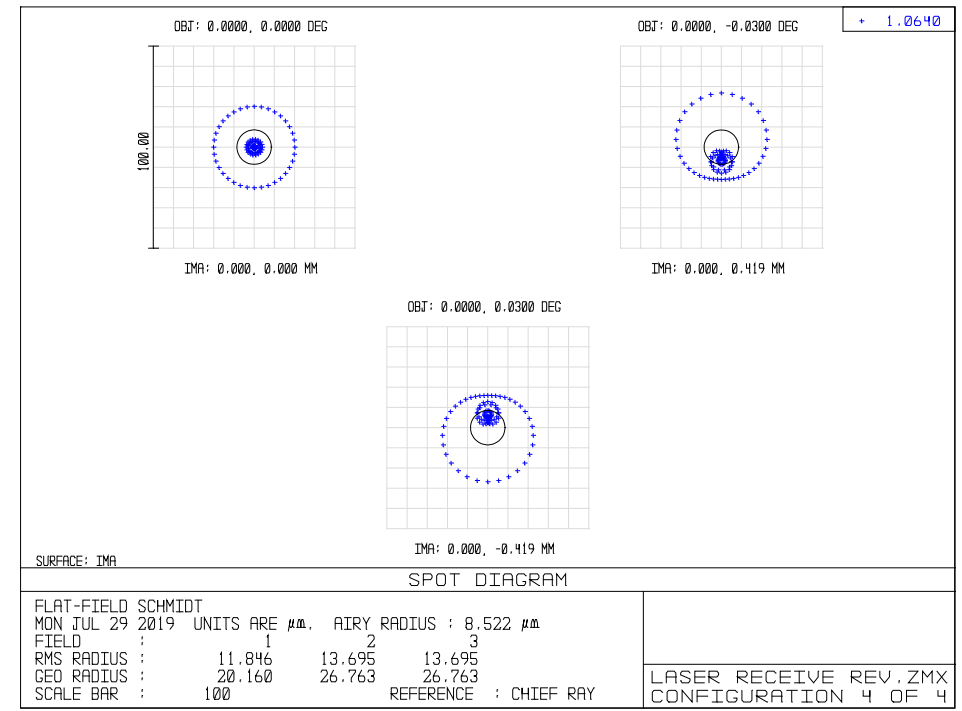

(c) $70^{\circ} \mathrm{C}$

图 2 激光接收光学系统点列图

\section{2）能量集中度}

能量集中度反映了光学系统各个视场在探测器 靶面上的能量分布情况。由光学设计软件得到激光接 收光学系统光斑能量随半径变化的分布曲线, 如图 3 所示。其中横坐标为以高斯像点为中心的包容圆半 径, 纵坐标为该包容圆所包含的能量。可以看出, 在 $20^{\circ} \mathrm{C} 、-50^{\circ} \mathrm{C}$ 和 $70^{\circ} \mathrm{C}$ 时, 激光接收全视场下, 能量集 中度高, 接近衍射极限。

\subsection{2 长波红外}

\section{1) 调制传递函数}

调制传递函数（Modulation Transfer Function, MTF）是红外光学系统像质评价最常用的评价指标, 它能全面定量地反映光学系统的衍射和像差所引起 的综合效应。图 4 为长波红外光学系统在 $20^{\circ} \mathrm{C} 、-50^{\circ} \mathrm{C}$

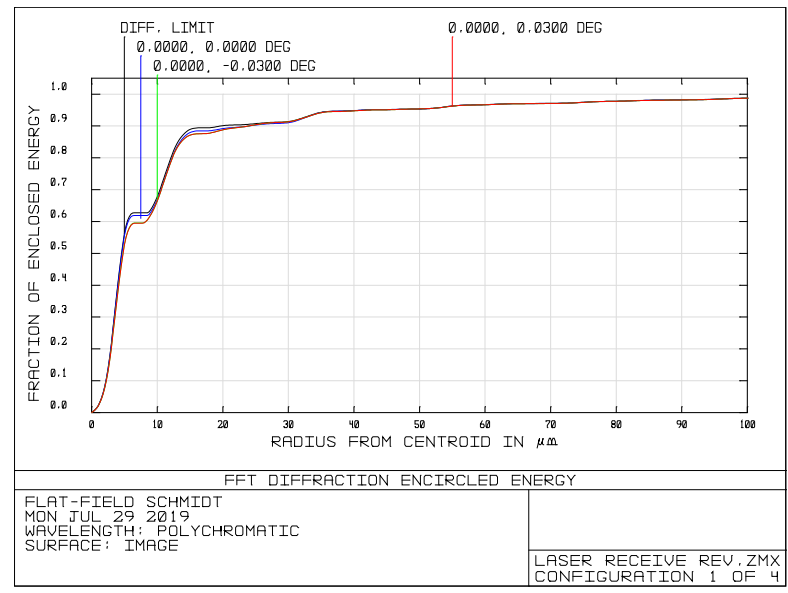

(a) $20^{\circ} \mathrm{C}$
和 $70^{\circ} \mathrm{C}$ 时各视场的 $\mathrm{MTF}$ 曲线。由图可见，长波红外 光学系统在 $-50^{\circ} \mathrm{C} \sim 70^{\circ} \mathrm{C}$ 范围内, 在奈奎斯特频率 17 $\mathrm{lp} / \mathrm{mm}$ 处，全视场内的 MTF 均大于 0.3 ; 中心视场的 MTF 值接近衍射极限, MTF 值大于 0.4 ; 边缘视场的 MTF 值大于 0.3 , 消热差后光学系统像质满足使用要 求。

\section{2) 点列图}

从图 5 可以看出, 长波红外光学系统在 $-50^{\circ} \mathrm{C} \sim$ $70^{\circ} \mathrm{C}$ 范围内, 中心视场的弥散斑半径均方根值, 小于 艾里斑半径, 达到衍射极限; 边缘视场的弥散斑半径 略大于艾里斑半径，接近衍射极限。消热差后，长波 红外光学系统成像质量良好, 弥散斑大部分都在一个 像元尺寸 $30 \mu \mathrm{m}$ 之内, 满足光学系统与红外探测器的 匹配要求。

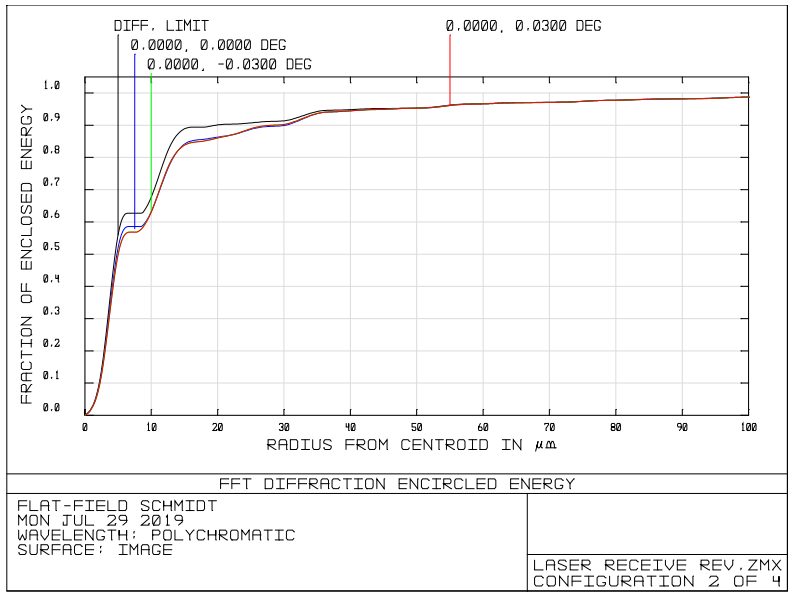

(b) $-50^{\circ} \mathrm{C}$ 


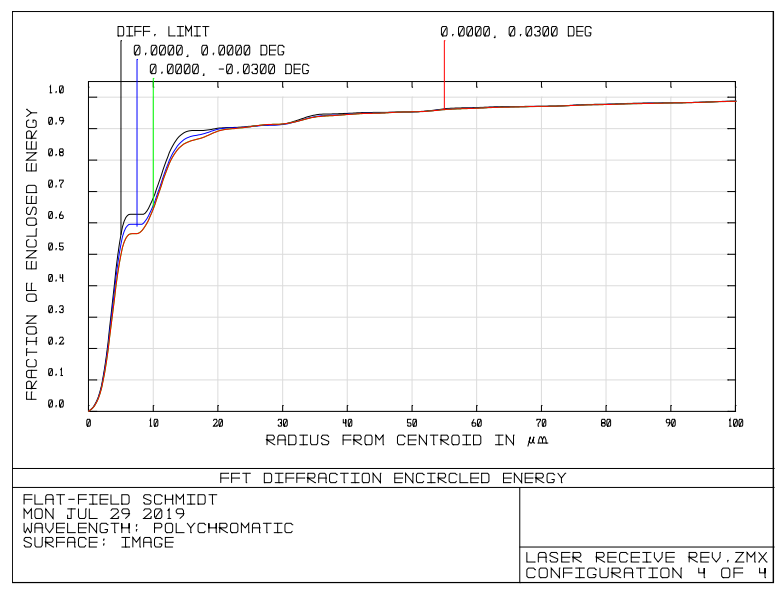

(c) $70^{\circ} \mathrm{C}$

图 3 激光接收光学系统能量集中度 Fig.3 Encircled energies of laser receiving optical system

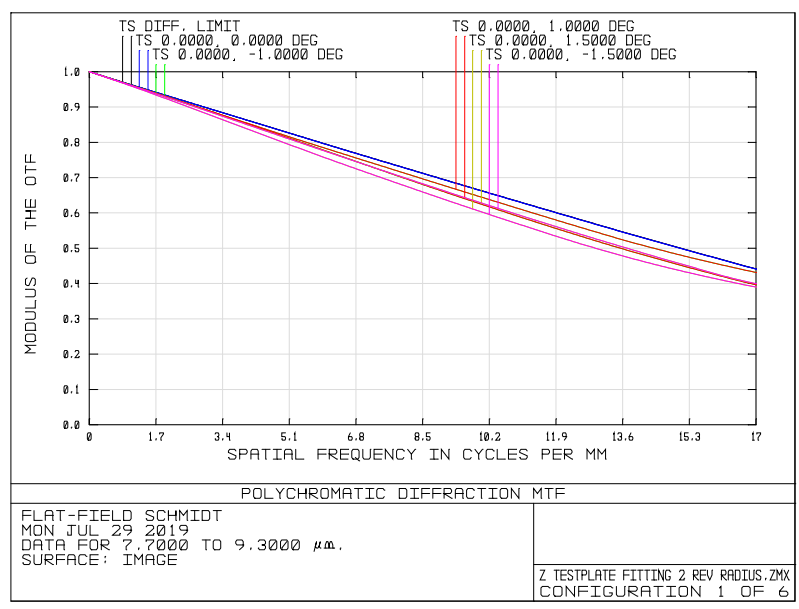

(a) $20^{\circ} \mathrm{C}$

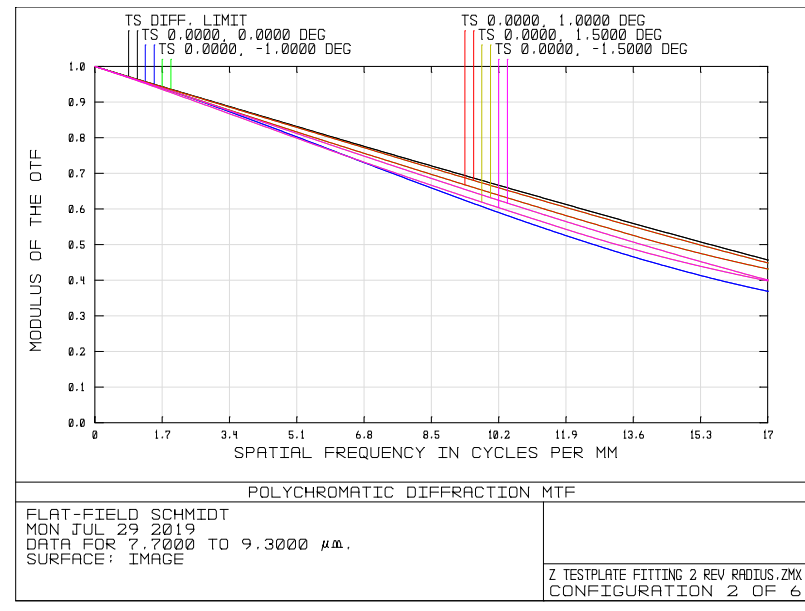

(b) $-50^{\circ} \mathrm{C}$

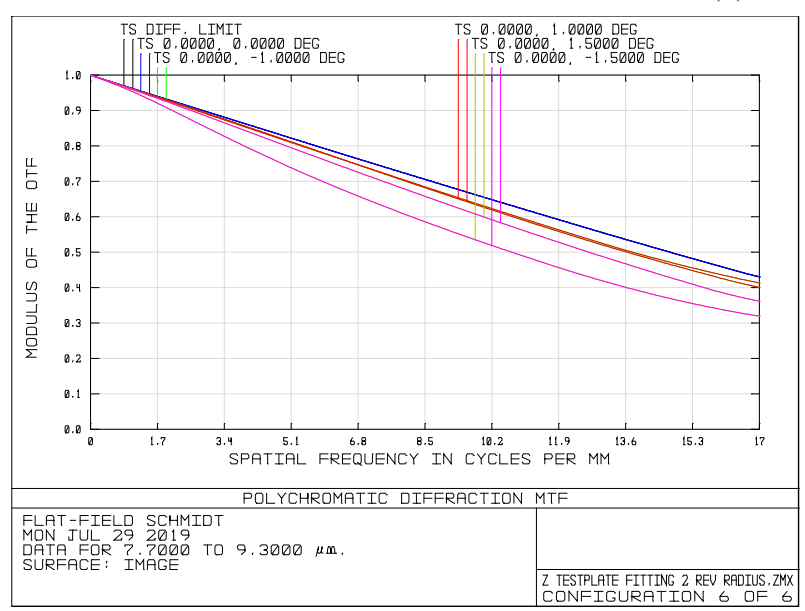

(c) $70^{\circ} \mathrm{C}$

图 4 长波红外光学系统 MTF

\section{3 结论}

基于红外搜索跟踪系统在激光测距和红外目标 探测方面的需求, 根据理论分析, 设计了一套适用于 $1.064 \mu \mathrm{m}$ 激光测距和 $320 \times 256$ 像元的长波红外凝视 焦平面阵列探测器的共孔径无热化紧凑型光学系统。
Fig.4 MTFs of LWIR optical system

该系统采用带有 $\mathrm{RC}$ 反射系统的折反式光学布局和 “川” 型结构, 实现了光机系统结构的紧凑性。光学 系统共用主镜和次镜, 利用次镜实现激光和红外分 光。长波红外采用二次成像结构, 达到 $100 \%$ 冷光阑 效率。通过合理分配光焦度、选择合适的光学材料和 结构材料、使用非球面等方法, 实现了光学被动式消 
热差。在 $-50^{\circ} \mathrm{C} \sim 70^{\circ} \mathrm{C}$ 环境温度下, $1.064 \mu \mathrm{m}$ 激光接 收光学系统各视场弥散斑均方根半径接近艾里斑半 径, 远小于 APD 探测器的敏感元直径 $0.8 \mathrm{~mm}$, 能量 集中度高; 7.7 9.3 $\mu \mathrm{m}$ 长波红外光学系统在奈奎斯特 频率 $17 \mathrm{lp} / \mathrm{mm}$ 处, 中心视场的 MTF 值大于 0.4 , 边缘 视场的 MTF 值大于 0.3 , 接近衍射极限, 成像性能良 好。该系统结构紧凑, 可应用于机载、车载等平台的 红外搜索、跟踪、识别和激光测距领域, 具有广阔的 应用前景。

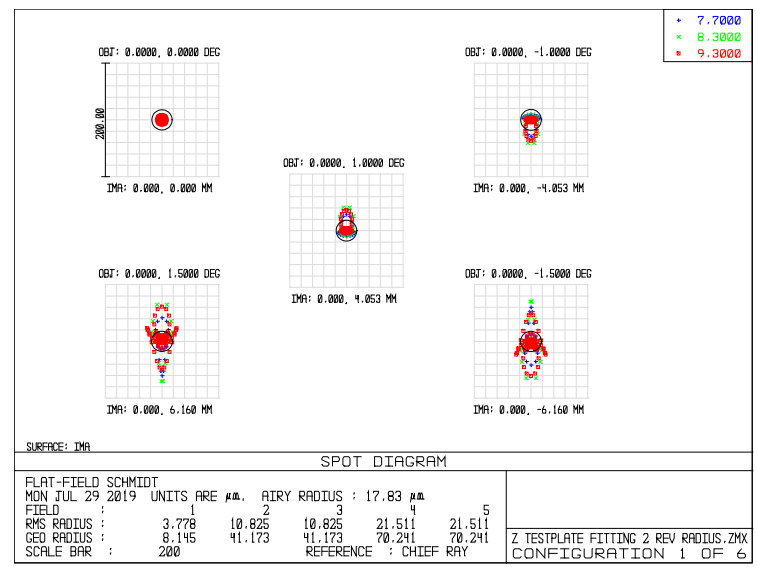

(a) $20^{\circ} \mathrm{C}$

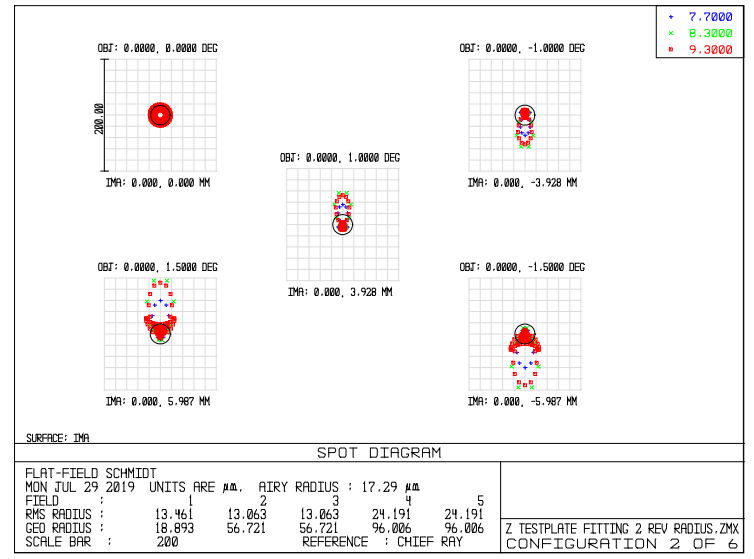

(b) $-50^{\circ} \mathrm{C}$

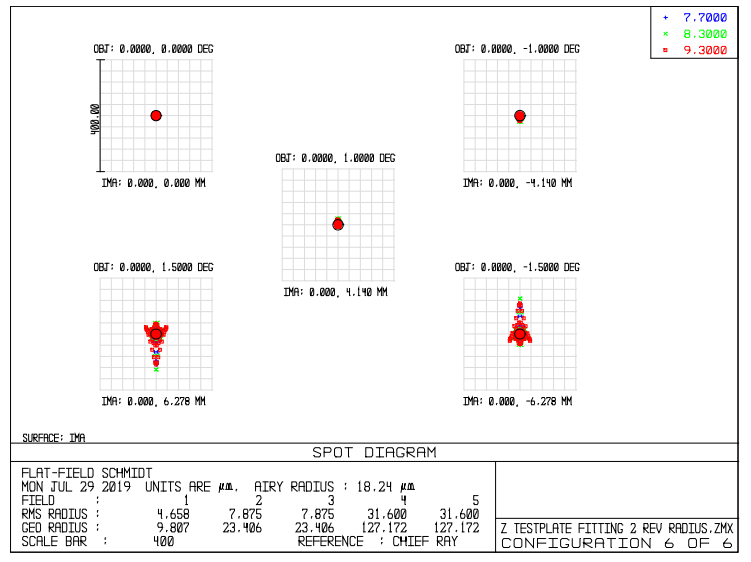

(c) $70^{\circ} \mathrm{C}$

图 5 长波红外光学系统点列图

Fig.5 Spot diagrams of LWIR optical system

\section{参考文献:}

[1] Jai Prakash Singh. An Algorithm for the Neural Fusion of IRST \& RADAR for Airborne Target Detection[C]//IEEE, 2005: 0-7803-93 23-6/05.

[2] 陈洁, 张若岗. 应用于红外搜索跟踪和态势感知系统的全景成像技术 [J]. 红外技术, 2016, 38(4): 269-279.

CHEN Jie, ZHANG Ruolan. Panoramic Imaging Technology Applied in IRST and Status Awareness System[J]. Infrared Technology, 2016, 38(4): 269-279.

[3] 钱昂, 何友金, 刘亮. 反舰导弹中波与长波红外成像制导优势对比研 究[J]. 红外技术, 2014, 36(8): 671-675.

QIAN Ang, HE Youjin, LIU Liang. A Comparative Study of the Advantage of Infrared Imaging Guidance Anti-ship Missiles Based on Medium Wave and Long-Wave[J]. Infrared Technology, 2014, 36(8): 671-675.

[4] 周子楠, 马军, 尉佩, 等. 激光/红外共光路无热化光学系统设计 [J]. 激光与光电子学进展, 2015, 52: 012202-1- 012202-7.

ZHOU Zinan, MA Jun, YU Pei, et al. Laser/Infrared Optical System Design of Common Optical Path and Athermalization[J]. Laser \& Optoelectronics Progress, 2015, 52: 012202-1- 012202-7.

[5] 沈庆云. 中波红外/激光双模导引头光学系统小型化设计 [D]. 哈尔滨: 哈尔滨工业大学, 2012.

SHEN Qingyun. Miniaturization Design of Optical System of Middle Infrared/Laser Dual-Mode Seeker[D]. Harbin: Harbin Institute of Technology, 2012.

[6] 姜波, 吴越豪, 戴世勋, 等. 紧凑型双波段无热化红外光学系统设计 [J]. 红外技术, 2015, 37(12): 999-1004.

JIANG Bo, WU Yuehao, DAI Shixun, et al. Design of a Compact Dual-band Athermalized Infrared System[J]. Infrared Technology, 2015, 37(12): 999-1004.

[7] 胡家升. 光学工程导论[M]. 2 版, 大连: 大连理工大学出版社, 2006: 802-804.

HU Jiasheng. Introduction to Optical Engineering[M]. Second Edition, Dalian: Dalian University of Technology Press, 2006: 802-804.

[8] McCulloch P M, Olson C. Eliminating dewar narcissus artifacts induced by moving optics in infrared staring focal plane sensors[C]//SPIE, 2012, 8468: 848606-848606N.

[9] 刘钧, 张胥斌, 高明. 制冷型中/长红外双波段双视场全景光学系统设 计 $[\mathrm{J}]$. 应用光学, 2016, 37(3): 456-464.

LIU Jun, ZHANG Xibin, GAO Ming. Design of cold MWIR/LWIR infrared dual-band/dual-field panoramic optical system[J]. Journal of Applied Optics, 2016, 37(3): 456-464.

[10] 李瑞瑶, 付跃刚, 刘智颖. 紧凑型中波红外成像系统无热化设计 [J]. 红外技术, 2018, 40(2):119-124.

LI Ruiyao, FU Yuegang, LIU Zhiying. Athermalization design of compact medium-wave infrared imaging system[J]. Infrared Technology, 2018, 40(2): 119-124.

[11] Mahajan V N. Optical Imaging and Aberrations: Part1, Ray Geometrical Optics[M]//SPIE, 1998.

[12] Jamieson $\mathrm{T}$ H. Athermalization of optical instruments from the optomechanical viewpoint[C]//SPIE, 1992(43): 131-159. 